山्山FANÇAISE

$>\mathrm{DE}$

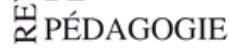

Revue française de pédagogie

Recherches en éducation

197 | 2016

Les multiples facettes de la créativité dans

l'apprentissage

GABATHULER Chloé. Apprécier la littérature. La relation esthétique dans l'enseignement de la lecture de textes littéraires

Rennes : Presses universitaires de Rennes, 2016, 274 p.

\title{
Agnès Perrin-Doucey
}

\section{(2) OpenEdition}

\section{Journals}

Édition électronique

URL : https://journals.openedition.org/rfp/5175

DOI : 10.4000/rfp.5175

ISSN : 2105-2913

Éditeur

ENS Éditions

Édition imprimée

Date de publication : 31 décembre 2016

Pagination : 138-140

ISSN : 0556-7807

Référence électronique

Agnès Perrin-Doucey, « GABATHULER Chloé. Apprécier la littérature. La relation esthétique dans

l'enseignement de la lecture de textes littéraires », Revue française de pédagogie [En ligne], 197| 2016, mis en ligne le 31 décembre 2016, consulté le 07 janvier 2022. URL : http://journals.openedition.org/rfp/ 5175 ; DOI : https://doi.org/10.4000/rfp.5175

Ce document a été généré automatiquement le 7 janvier 2022

(c) tous droits réservés 


\title{
GABATHULER Chloé. Apprécier la littérature. La relation esthétique dans l'enseignement de la lecture de textes littéraires
}

Rennes : Presses universitaires de Rennes, 2016, 274 p.

\author{
Agnès Perrin-Doucey
}

\section{RÉFÉRENCE}

GABATHULER Chloé. Apprécier la littérature. La relation esthétique dans l'enseignement de la lecture de textes littéraires. Rennes : Presses universitaires de Rennes, 2016, 274 p.

1 Un ouvrage qui devrait faire date affirme Bertrand Daunay en conclusion de sa préface. En effet, cette contribution originale aux recherches conduites par les didacticiens de la littérature mérite une lecture attentive car elle ouvre, avec précision et conviction, une réflexion inédite. Savoir rendre compte de la forme prise par la relation esthétique de l'élève aux textes littéraires en contexte scolaire et du rôle qui lui est conféré dans la lecture, être alors à même d'esquisser des pistes didactiques et des outils pour favoriser son émergence et son partage en classe, telles sont les ambitions de l'auteure. Dans les deux premières parties, Chloé Gabathuler prend le temps d'une large réflexion théorique puis méthodologique, sur laquelle nous allons revenir, qui confère à son propos la rigueur et l'éthique indispensables à la construction de savoirs didactiques nouveaux par la recherche. La troisième partie est consacrée à l'analyse d'un recueil de "pratiques effectives" de la lecture littéraire, établi minutieusement par l'équipe GRAFE de l'Université de Genève. La synthèse qui clôt cette analyse permet de questionner les résultats obtenus pour ouvrir en conclusion des pistes de transformation des pratiques et des perspectives pour les recherches à venir. 
2 La première partie fonde - au sens propre du terme - un cadre théorique autour de la notion de lecture littéraire à l'école qui a le mérite de revisiter les travaux de quelques littéraires (Todorov, Schaeffer, Citton) et de didacticiens issus du monde francophone à l'aune d'une réflexion sur la relation esthétique comme modalité de réception des textes.

3 Le premier chapitre interroge les enjeux didactiques qu'implique une définition de la lecture comme expérience et la notion de sujet-lecteur construite par les didacticiens. Il met en évidence et discute les jeux de subjectivation qu'impose l'expérience lectrice et de dé-subjectivation qu'impliquent la pratique scolaire et ses processus de modélisation des savoirs connexes à la nécessaire transposition didactique. Ainsi sont questionnées à nouveau, au moins dans l'implicite de l'argumentation, les divergences scientifiques dans la description des notions de sujet-lecteur et de sujet-didactique. Mais Chloé Gabathuler ne se contente pas de décrire, elle se positionne sans ambiguïté en se tournant résolument du côté du sujet-didactique pour analyser les traces du travail et de la réception esthétique de l'élève-lecteur en formation.

Dans le second chapitre, l'auteure revient sur l'idée de relation esthétique pour interroger en premier lieu les distinctions philosophiques entre jugement et appréciation, entre éthique et esthétique; puis elle recentre le propos sur le fonctionnement esthétique des textes. Le chapitre débouche sur l'énoncé d'une conception personnelle de la relation esthétique, organisée autour des notions d'appréciation et d'évaluation et qui s'installe dans une triangulation entre individu, collectivité et système de jugements. Il engage donc l'idée, qui sera reprise tout au long de l'ouvrage, que le contexte de scolarisation des textes et des lectures modifie nécessairement les pratiques sociales d'appréciation ou d'évaluation des œuvres alors même que les locuteurs semblaient vouloir les reproduire. L'auteure justifie ainsi la nécessité de penser didactiquement la relation esthétique avec les textes en contexte scolaire.

5 De ce fait, elle conduit habilement son lecteur vers un troisième chapitre où est alors présentée la notion de transposition didactique appliquée au concept étudié. S'appuyant sur les travaux de Chevallard et sur leur reconfiguration par Schneuwly et Ronveaux qui introduisent une réflexion sur l'objet enseigné, la chercheuse expose alors la nécessité et l'intérêt de confronter son objet de recherche aux pratiques effectives de lecture littéraire. Dans cette perspective, elle analyse aussi avec précision le Plan d'études romand déterminant le contexte institutionnel de son expérimentation.

6 À l'issue de cette partie, une thèse est formulée entre trois points majeurs :

- la relation esthétique se manifeste dans des jugements esthétiques, éthiques et émotionnels ;

- les modalités de jugements se modifient avec l'évolution des niveaux, partant de jugements émotionnels pour aller vers des jugements esthétiques ;

- les jugements ne sont pas considérés comme objet d'enseignement et se présentent dans les pratiques de manière sédimentée, ils évoluent en fonction des textes, des niveaux et au cours des séances.

7 La seconde partie, beaucoup plus brève, présente le protocole expérimental et les choix méthodologiques réalisés. La recherche s'appuie sur un corpus de données recueillies dans plusieurs classes de primaire et secondaire et mutualisé par les chercheurs du GRAFE engagés dans le projet. Il s'agit de comparer les approches de deux œuvres. L'une («Le loup et l'agneau» de Jean de la Fontaine) est choisie pour sa dimension 
classique et parce qu'il existe un important corpus didactique et pédagogique; la seconde ("La négresse et le chef des avalanches» de Jean Lovay) est choisie au contraire parce qu'elle est absente de «la vulgate scolaire » (p. 103). Deux séances sont filmées dans chacune des 30 classes du panel ( $6^{\mathrm{e}}$ primaire, $8^{\mathrm{e}}$ secondaire I et $2^{\mathrm{e}} \mathrm{du}$ secondaire II) et les traces des activités données aux élèves sont recueillies dans un classeur. L'analyse veut croiser deux formes de variations relatives aux objets textuels d'une part, aux niveaux d'enseignement d'autre part. Enfin, les niveaux d'analyse sont décrits minutieusement. Ils concernent essentiellement le jugement et ses catégorisations, les traces et le contenu des interactions, le rôle des enseignants dans les interactions.

8 La troisième partie est consacrée à l'analyse. Étayée par un recours permanent aux retranscriptions minutieuses du corpus, l'analyse s'organise en quatre chapitres. Le premier se consacre à la relation esthétique quand elle fait l'objet d'activités scolaires dans 28 leçons sur les $60 \mathrm{du}$ panel. Celle-ci relève de l'oral et est repérée dans « des réactions à chaud » qui semblent médiatisées par la reformulation du sens littéral du texte.

9 Le second chapitre interroge la notion de jugements dans la lecture des textes. La double analyse, quantitative et qualitative, des occurrences de jugements indique d'une part une présence continue et progressive en fonction des niveaux d'enseignement, d'autre part une relation esthétique qui se développe par l'interprétation.

10 Puis vient une analyse des interactions pour comprendre comment se construit la relation esthétique. La présence d'interactions entre pairs, de fortes variations liées aux niveaux d'enseignement mais aussi au choix du texte est mise en évidence. Les interactions apparaissent comme plus rares et moins argumentées lors de la lecture de la fable mais aussi avec la nouvelle de Lovay au secondaire II. La dimension sociale des travaux de groupes semble propice aux échanges et à "la coopération dialogale et argumentative » (p. 205).

11 Le dernier chapitre et la conclusion reviennent sur l'ensemble de l'analyse et valident les hypothèses de recherche. Enfin, pour conclure, Chloé Gabathuler propose des pistes de recherches complémentaires de type «ingénierie didactique» (p. 223) : interroger l'appropriation de l'œuvre et les jugements, analyser les traces écrites de la relation esthétique, le travail des enseignants englobant la progressivité des apprentissages, le choix des textes et l'évaluation. Elle réaffirme ainsi la nécessité de concevoir didactiquement la relation esthétique, quand bien même celle-ci est présente sous des formes diverses dans la lecture littéraire quel que soit le niveau de lecture.

12 L'ouvrage de Chloé Gabathuler interroge donc avec méthode et finesse une composante essentielle parce que transversale de la lecture littéraire, jusqu'ici impensée didactiquement. Fidèle à l'esprit de la collection "Paideia ", il s'inscrit comme une référence utile pour les didacticiens de la littérature comme du français, chercheurs ou formateurs. 


\section{AUTEURS}

\section{AGNÈS PERRIN-DOUCEY}

Université de Montpellier, faculté d'Éducation 